\title{
Effect on Jarlskog Determinant Above the GUT Scale Within Four Flavor Neutrino Framework
}

\author{
Vivek Kumar Nautiyal ${ }^{1}$ (D) Bipin Singh Koranga ${ }^{2}$ (D)
}

Received: 2 June 2021 / Accepted: 22 July 2021 / Published online: 12 August 2021

(C) The Author(s) 2021

\begin{abstract}
We study the Planck scale effects on Jarlskog determiant in the four flavor framework. On electroweak symmetry breaking, quantum gravitational effects lead to an effective SU(2) $\times \mathrm{U}(1)$ invariant dimension-5 Lagrangian including neutrino and Higgs forces, which perturbed the neutrino mass term and produce an extra terms in the neutrino mass matrix. We consider that gravitational interaction is independent from flavor and compute the Jarlskog determiant due to Planck scale effects. In the case of leptonic sector, the strentgh of CP violation is measured by Jarlskog determiant. We applied our approach to study Jarlskog determinant in the four flavor neutrino mixing above the GUT scale.
\end{abstract}

Keywords Jarlskog determinant · CP violaton · Planck scale

\section{Introduction}

The evidence of a deficit of detected solar neutrinos [1] indicates that electron neutrinos must also participate in lepton mixing. Recently from the analysis of the Super-Kamiokande data [2], the normal (inverted) neutrino mass squared differences with $90 \%$ CL were found to be $1.9(1.7) \times 10^{-3} \mathrm{eV}^{2} \leq \Delta_{31} \leq 2.6(2.7) \times 10^{-3} \mathrm{eV}^{2}$. The parameters of sterile neutrino (mass squared difference $\Delta_{41}=m_{4}^{2}-m_{i}^{2}$ and the mixing angle $\left(\sin ^{2} \theta_{14}\right)$ ) are excluded by the joint results of Day Bay, MINOS and Bugey-3 data [3]. The latest best fit value of neutrino mass square difference and mixing angle from the analysis of global neutrino oscillation data are found to be [2] $\Delta_{41}=1.7 \mathrm{eV}^{2}$ and $\sin ^{2} \theta_{14}=0.019$. In search of neutrino oscillation, three different scale of neutrino mass-squared differences $\triangle m_{\text {sun }}^{2} \ll \Delta m_{\text {atm }}^{2} \ll \Delta m_{L S N D}^{2}$ obtained by atmospheric and solar neutrino oscillation experiments and LSND collabration, which required four netrinos with definite mass to explain these data. Super- Kamiokande Collaboration, Y. Fukuda et al. [4] experimenatal data was in good agreement with two flavor oscillation i.e. $v_{\mu} \rightarrow v_{\epsilon}$ and provided evidence for neutrino oscillation. Another Liquid Scientillator Neutrino Detector (LSND) also

Bipin Singh Koranga

bipiniitb@rediffmail.com

1 Department of Physics, Babasaheb Bhimrao Ambedkar University, Lucknow, 226025, India

2 Department of Physics, Kirori Mal college (University of Delhi), Delhi, 110007, India 
confirmed atmospheric neutrino oscillation $v_{\mu} \rightarrow v_{\epsilon}$ through muon neutrino flux from $\Pi^{\dot{+}}$ decay in flight (DIF) which has completely different backgrounds from the $\bar{v}_{\mu} \rightarrow \bar{v}_{\epsilon}$ DAR oscillation search . LSND experiment provided that both effect was due to neutrino oscillation by C. Athanassopoulos et al. [5]. All these neutrino oscillation data could be explained successfully by four neutrinos with definite mass by S.M. Bilenky et al. [6] .Thus four-neutrino models have been studied by many authors [10-13].

The assumption of the violation of $\mathrm{CP}$ and $\mathrm{T}$ symmetries are to be profound in the four-neutrino mixing models. The modern long oscillation length accelerator neutrino experiments are needed for the observation of leptonic side $\mathrm{CP}$ and T-violation. There are many ways to incorporated the non trivial CP violating complex phases in $4 \mathrm{x} 4$ lepton flavor mixing matrix. These relative phases and magnitude of the coupling consants are responsible for maximal CP violation. For real mixing matrix there is no CP symmetry violation, but in case of neutrino flavor mass mixing matrices some rephasing Jarlskog invariants are assigned for $\mathrm{CP}$ and $\mathrm{T}$ violation by $\mathrm{C}$. Jarlskog [7] . Within the framework of four-neutrino mixing, the role of these Jarlskog invariants are very much crucial for the study of violation in $\mathrm{CP}$ and $\mathrm{T}$.

This paper is organized as follows. In Section 2, we briefly describe the Jarlskog invariants which leads to an $\mathrm{CP}$ violation. In Section 3 we summarize the effect of planck scale for four neutrino mixing angle. We also investigate the effect of these planck scale on Jarlskog determinant in Section 4. Results and Conclusions are briefly discussed in Sections 5 and 6 respectively.

\section{Jarlskog Determinant in Four Flavor Framework}

$\mathrm{CP}$ violation arise as three or more generation $[8,9] . \mathrm{CP}$ violation in neutrino oscillation is interesting because it relates directly to $\mathrm{CP}$ phase parameter in the mixing for $n_{i} 3$ degenerate neutrino. We can write down the compact formula for the difference of transition probability between conjugate channel.

$$
\begin{gathered}
\Delta P_{\alpha \beta}=P\left(v_{\alpha} \rightarrow v_{\beta}\right)-P\left(v_{\beta} \rightarrow v_{\alpha}\right) \\
P\left(v_{\alpha} \rightarrow v_{\beta}\right)=\delta_{\alpha \beta}-\sum_{i<j}\left[4 \operatorname{Re}\left(X_{\alpha \beta}^{i j}\right) \sin ^{2} \Delta_{i j}-2 \operatorname{Im}\left(X_{\alpha \beta}^{i j}\right) \sin 2 \Delta_{i j}\right]
\end{gathered}
$$

where,

$$
\begin{gathered}
(\alpha, \beta)=(s, e),(\tau, s),(e, \mu) \\
X_{\alpha \beta}^{i j}=\left(U_{\alpha i} U_{\beta j} U_{\alpha j}^{*} U_{\beta i}^{*}\right) \\
\Delta_{i j}=\frac{\delta m_{i j}^{2} L}{4 E}, \delta m_{i j}^{2}=m_{i}^{2}-m_{j}^{2},
\end{gathered}
$$

$\mathrm{L}$ is the oscillation distance and $\mathrm{E}$ is the neutrino energy. The quantity $X_{\alpha \beta}^{i j}[14]$ are related to the Jarlskog invariant by [15]

$$
J_{\alpha \beta}^{i j} \approx \operatorname{Im}\left(X_{\alpha \beta}^{i j}\right)
$$

The goal of ongoing experiments are the determination of the upper bound on the parameters of four neutrino mixing angles. In particularly, the observation of $\delta$ is quites interesting for 
the point of view that $\delta$ related to the origin of the matter in the universe. The determination of $\delta$ is the important goal of the experiments.

As we know that sterile neutrino do not interact with the weak interaction, they might be oscillate with active neutrinos. Once consider the sterile neutrino we are led to a more general $\left(n+n^{\prime}\right) \times\left(n+n^{\prime}\right)$ lepton flavor mixing matrix [16], defined as $U$ in the chosen flavor basis. For the flavor mixing of one sterile neutrino $\left(v_{\mathrm{s}}\right)$ and three active neutrinos $\left(v_{\mathrm{e}}, v_{\mu}, v_{\tau}\right)$, the explicit form of $\mathrm{U}[10]$ can be written as

$$
U=\left(\begin{array}{cccc}
U_{s 1} & U_{s 2} & U_{s 3} & U_{s 4} \\
U_{e 1} & U_{e 2} & U_{e 3} & U_{e 4} \\
U_{\mu 1} & U_{\mu 2} & U_{\mu 3} & U_{\mu 4} \\
U_{\tau 1} & U_{\tau 2} & U_{\tau 3} & U_{\tau 4}
\end{array}\right)
$$

The matrix $U$ contains six mixing angles and three phase angles for Dirac neutrinos and for Majorana neutrinos, We required three additional phase angles for proper defining the oscillation matrix $\mathrm{U}$. The rephasing invariants Jarlskog [7] of CP or T violation for both cases are as follows :

$$
J_{\alpha \beta}^{i j} \approx \operatorname{Im}\left(U_{\alpha i} U_{\beta j} U_{\alpha j}^{*} U_{\beta i}^{*}\right)
$$

where, $(\alpha, \beta=\mathrm{s}, \mathrm{e}, \mu, \tau)$ and $(i=1,2,3,4)$.

Since $\mathrm{U}$ is Unitary matrix, Thus we get,

and

$$
J_{\alpha \beta}^{i i}=J_{\alpha \alpha}^{i j}=J_{\beta \beta}^{i j}=J_{\alpha \beta}^{j j}=0
$$

$$
J_{\alpha \beta}^{i j}=-J_{\alpha \beta}^{j i}=J_{\beta \alpha}^{i j}=-J_{\beta \alpha}^{j i}
$$

Hence we deals with four flavor framework by incorporating the sterile neutrino of $\mathrm{eV}$ range and the mixing of this sterile neutrino with three neutrinos is light. By adding one sterile neutrinos [11], there is an increment in mixing angles and CP violating phases in the PMNS matrix $U_{4 \times 4}$ which is given by,

$$
U=R_{34} R_{24} R_{14} R_{23} R_{13} R_{12},
$$

where the matrices $R_{i j}$ are rotations in ij space,

$$
R_{12}=\left(\begin{array}{cccc}
c_{12} & s_{12} e^{-i \delta_{12}} & 0 & 0 \\
-s_{12} e^{i \delta_{12}} & c_{12} & 0 & 0 \\
0 & 0 & 1 & 0 \\
0 & 0 & 0 & 1
\end{array}\right) \quad \text { and } \quad R_{34}=\left(\begin{array}{cccc}
1 & 0 & 0 & 0 \\
0 & 1 & 0 & 0 \\
0 & 0 & c_{34} & s_{34} \\
0 & 0 & -s_{34} & c_{34}
\end{array}\right)
$$

where $s_{i j}=\sin \theta_{i j} e^{i \delta_{i j}}, c_{i j}=\cos \theta_{i j}$.

Note that there are in total six CP-violating phase $\delta_{i j}$. The explicit form of $\mathrm{U}$ is

$$
\begin{gathered}
U_{s 1}=\left(c_{14} c_{13} c_{12}\right), \\
U_{s 2}=\left(c_{14} c_{13} s_{12} e^{-i \delta_{12}}\right), \\
U_{s 3}=\left(c_{14} s_{13} e^{-i \delta_{13}}\right), \\
U_{s 4}=\left(s_{14} e^{-i \delta_{14}}\right), \\
U_{e 1}=\left(-c_{24} c_{23} s_{12} e^{i \delta_{12}}-c_{24} s_{23} e^{-i \delta_{23}} s_{13} e^{i \delta_{13}} c_{12}-s_{24} e^{-i \delta_{24}} s_{14} e^{i \delta_{14}} c_{13} c_{12}\right),
\end{gathered}
$$




$$
\begin{aligned}
& U_{e 2}=\left(c_{24} c_{23} c_{12}-c_{24} s_{23} e^{-i \delta_{23}} s_{13} e^{i \delta_{13}} s_{12} e^{-i \delta_{12}}-s_{24} e^{-i \delta_{24}} s_{14} e^{i \delta_{14}} c_{13} s_{12} e^{-i \delta_{12}}\right), \\
& U_{e 3}=\left(c_{13} c_{24} s_{23} e^{-i \delta_{23}}-s_{24} e^{-i \delta_{24}} s_{14} e^{i \delta_{14}} s_{13} e^{-i \delta_{13}}\right), \\
& U_{e 4}=\left(c_{14} S_{24} e^{-i \delta_{24}}\right), \\
& U_{\mu 1}=\left(c_{34} s_{23} e^{i \delta_{23}} s_{12} e^{i \delta_{12}}-c_{34} c_{23} s_{13} e^{i \delta_{13}} c_{12}+s_{34} e^{-i \delta_{34}} s_{24} e^{i \delta_{24}} c_{23} s_{12} e^{i \delta_{12}}\right. \\
& +s_{34} e^{-i \delta_{34}} s_{24} e^{i \delta_{24}} s_{23} e^{-i \delta_{23}} s_{13} e^{i \delta_{13}} c_{12} \\
& \left.-s_{34} e^{-i \delta_{34}} c_{24} s_{14} e^{i \delta_{14}} c_{13} c_{12}\right) \text {, } \\
& U_{\mu 2}=\left(-c_{34} s_{23} e^{i \delta_{23}} c_{12}-c_{34} c_{23} s_{13} e^{-i \delta_{13}} s_{12} e^{i \delta_{12}}-s_{34} e^{-i \delta_{34}} s_{24} e^{i \delta_{24}} c_{23} c_{12}\right. \\
& +s_{34} e^{-i \delta_{34}} s_{24} e^{i \delta_{24}} s_{23} e^{-i \delta_{23}} s_{13} e^{i \delta_{13}} s_{12} e^{-i \delta_{12}} \\
& \left.-s_{34} e^{-i \delta_{34}} c_{24} s_{14} e^{i \delta_{14}} c_{13} s_{12} e^{-i \delta_{12}}\right) \text {, } \\
& U_{\mu 3}=\left(c_{34} c_{23} c_{13}-s_{34} e^{-i \delta_{34}} s_{24} e^{i \delta_{24}} s_{23} e^{-i \delta_{23}} c_{13}-s_{34} e^{-i \delta_{34}} c_{24} s_{14} e^{i \delta_{14}} s_{13} e^{-i \delta_{13}}\right) \\
& U_{\mu 4}=\left(s_{34} e^{-i \delta_{34}} c_{24} c_{14}\right) \\
& U_{\tau 1}=\left(s_{34} s_{12} s_{23}+s_{34} e^{i \delta_{34}} c_{23} s_{13} e^{i \delta_{14}} c_{12}+c_{34} s_{24} e^{i \delta_{24}} c_{23} s_{12} e^{i \delta_{12}}\right. \\
& +c_{34} s_{24} e^{i \delta_{34}} s_{23} e^{-i \delta_{23}} s_{12} e^{i \delta_{12}} c_{12} \\
& \left.-c_{34} c_{24} s_{14} e^{i \delta_{14}} c_{13} c_{12}\right) \text {, } \\
& U_{\tau 2}=\left(s_{34} e^{i \delta_{34}} c_{12} s_{23} e^{i \delta_{23}}+s_{34} e^{i \delta_{34}} c_{23} s_{13} e^{i \delta_{13}} s_{12} e^{-i \delta_{12}}-c_{34} s_{24} e^{i \delta_{24}} c_{23} c_{12}\right. \\
& +c_{34} s_{24} e^{i \delta_{34}} s_{23} e^{-i \delta_{23}} s_{13} e^{i \delta_{13}} s_{12} e^{-i \delta_{12}} \\
& \left.-c_{34} c_{24} s_{14} e^{i \delta_{14}} c_{13} s_{12} e^{-i \delta_{12}}\right) \text {, } \\
& U_{\tau 3}=\left(-s_{34} e^{i \delta_{34}} c_{23} c_{13}-c_{34} s_{24} e^{i \delta_{24}} s_{23} e^{-i \delta_{23}} c_{13}-c_{34} c_{24} s_{14} e^{i \delta_{14}} s_{13} e^{-i \delta_{13}}\right) \\
& U_{\tau 4}=\left(c_{34} c_{24} c_{14}\right) \text {, }
\end{aligned}
$$

We get the analytical expression for $P(\alpha, \beta)$ using the usual form of the MNS matrix parametrization given by $\mathrm{U}$,

$$
\begin{aligned}
P\left(v_{\alpha} \rightarrow v_{\beta}\right)= & \delta_{\alpha \beta}-\left[4 \left\{\operatorname{Re}\left(X_{\alpha \beta}^{12}\right) \sin ^{2} \Delta_{12}+\operatorname{Re}\left(X_{\alpha \beta}^{13}\right) \sin ^{2} \Delta_{13}+\operatorname{Re}\left(X_{\alpha \beta}^{14}\right) \sin ^{2} \Delta_{14}\right.\right. \\
& \left.+\operatorname{Re}\left(X_{\alpha \beta}^{23}\right) \sin ^{2} \Delta_{23}+\operatorname{Re}\left(X_{\alpha \beta}^{24}\right) \sin ^{2} \Delta_{24}+\operatorname{Re}\left(X_{\alpha \beta}^{34}\right) \sin ^{2} \Delta_{34}\right\} \\
& -2\left\{J_{\alpha \beta}^{12} \sin 2 \Delta_{12}+J_{\alpha \beta}^{13} \sin 2 \Delta_{13}+J_{\alpha \beta}^{14} \sin 2 \Delta_{14}\right. \\
& \left.\left.+J_{\alpha \beta}^{23} \sin 2 \Delta_{23}+J_{\alpha \beta}^{24} \sin 2 \Delta_{24}+J_{\alpha \beta}^{34} \sin 2 \Delta_{34}\right\}\right]
\end{aligned}
$$

We get nine independent [24] $J_{\alpha \beta}^{i j}$, whose magnitudes depends only upon three of the six CP-violating phases or their combinations in a specific parametrization of $U$. The nine 
jarlskog $J_{\alpha \beta}^{i j}$ and six mixing angles for four flavour mixing are define using the matrix $\mathrm{U}$ in (4) are,

$$
\begin{aligned}
& J_{s e}^{13}=\operatorname{Im}\left(U_{s 1} U_{e 3} U_{s 3}^{*} U_{e 1}^{*}\right) \\
& J_{s e}^{24}=\operatorname{Im}\left(U_{s 2} U_{e 4} U_{s 4}^{*} U_{e 2}^{*}\right) \\
& J_{s e}^{34}=\operatorname{Im}\left(U_{s 3} U_{e 4} U_{s 4}^{*} U_{e 3}^{*}\right) \\
& J_{\tau s}^{13}=\operatorname{Im}\left(U_{\tau 1} U_{s 3} U_{\tau 3}^{*} U_{s 1}^{*}\right) \\
& J_{\tau s}^{14}=\operatorname{Im}\left(U_{\tau 1} U_{s 4} U_{\tau 4}^{*} U_{s 1}^{*}\right) \\
& J_{\tau s}^{34}=\operatorname{Im}\left(U_{\tau 3} U_{s 4} U_{\tau 4}^{*} U_{s 3}^{*}\right) \\
& J_{e \mu}^{23}=\operatorname{Im}\left(U_{e 2} U_{\mu 3} U_{e 3}^{*} U_{\mu 2}^{*}\right) \\
& J_{e \mu}^{24}=\operatorname{Im}\left(U_{e 2} U_{\mu 4} U_{e 4}^{*} U_{\mu 2}^{*}\right) \\
& J_{e \mu}^{34}=\operatorname{Im}\left(U_{e 3} U_{\mu 4} U_{e 4}^{*} U_{\mu 3}^{*}\right)
\end{aligned}
$$

And,

$$
\begin{gathered}
\sin ^{2} \theta_{14}=\left|\mathrm{U}_{\mathrm{s} 4}\right|^{2} \\
\sin ^{2} \theta_{24}=\frac{\left|\mathrm{U}_{\mathrm{e} 4}\right|^{2}}{1-\left|\mathrm{U}_{\mathrm{s} 4}\right|^{2}} \\
\sin ^{2} \theta_{34}=\frac{\left|\mathrm{U}_{\mu 4}\right|^{2}}{1-\left|\mathrm{U}_{\mathrm{s} 4}\right|^{2}-\left|\mathrm{U}_{\mathrm{e} 4}\right|^{2}} \\
\sin ^{2} \theta_{13}=\frac{\left|\mathrm{U}_{\mathrm{s} 3}\right|^{2}}{1-\left|\mathrm{U}_{\mathrm{s} 4}\right|^{2}} \\
\sin ^{2} \theta_{12}=\frac{\left|\mathrm{U}_{\mathrm{s} 2}\right|^{2}}{1-\left|\mathrm{U}_{\mathrm{s} 4}\right|^{2}-\left|\mathrm{U}_{\mathrm{s} 3}\right|^{2}} \\
\sin ^{2} \theta_{23}=\frac{\left|\mathrm{U}_{\mathrm{e} 3}\right|^{2}\left(1-\left|\mathrm{U}_{\mathrm{s} 4}\right|^{2}\right)-\left(\left|\mathrm{U}_{\mathrm{s} 4}\right|^{2}\left|\mathrm{U}_{\mathrm{e} 4}\right|^{2}\right)}{1-\left|\mathrm{U}_{\mathrm{s} 4}\right|^{2}-\left|\mathrm{U}_{\mathrm{e} 4}\right|^{2}} \\
+\frac{\left|U_{s 1} U_{e 1}+U_{s 2} U_{e 2}\right|^{2}\left(1-\left|U_{s 4}\right|^{2}\right)}{\left(1-\left|U_{s 4}\right|^{2}-\left|U_{s 3}\right|^{2}\right)\left(1-\left|U_{s 4}\right|^{2}-\left|U_{e 4}\right|^{2}\right)}
\end{gathered}
$$

Equation (10) is very much crucial to study the role of violation in $\mathrm{CP}$ and $\mathrm{T}$ within the framework of four-neutrino mixing. B. S. Koranga et. al. [17-19, 22, 23] did some analysis on $\mathrm{CP}$ and $\mathrm{T}$ violation within two or three flavor framework for different parameterization and above GUT scale.

\section{Four Neutrino Mixing Angles Above the GUT Scale}

Neutrino mass square differences and mixing angles above the GUT scale are developed in paper [21]. A neutrino mass matrix $M$ is given by

$$
\mathbf{M}=U^{*} \operatorname{diag}\left(M_{i}\right) U^{\dagger},
$$


where, $U_{\alpha i}$ is the usual mixing matrix and $M_{i}$, the neutrino masses is generated by Grand unified theory. Most of the parameter related to neutrino oscillation are known, the major expectation is given by the mixing elements $U$.

In term of the above mixing angles, the mixing matrix is

$$
U=\operatorname{diag}\left(e^{i f 1}, e^{i f 2}, e^{i f 3}, e^{i f 4}\right) R\left(\theta_{34}\right) R\left(\theta_{24}\right) \Delta R\left(\theta_{14}\right) \Delta^{*} R\left(\theta_{23}\right) R\left(\theta_{13}\right) R\left(\theta_{12}\right) \operatorname{diag}\left(e^{i \alpha}, e^{i \beta}, e^{i \gamma}, 1\right)
$$

The matrix $\Delta=\operatorname{diag}\left(e^{\frac{i \delta}{2}}, 1,1, e^{\frac{-i \delta}{2}}\right)$ contains the Dirac phase. This leads to CP violation in neutrino oscillation $\alpha, \beta$ and $\gamma$ are the so called Majorana phases, which effects the neutrinoless double beta decay. $f_{1}, f_{2}, f_{3}$ and $f_{4}$ are usually absorbed as a part of the definition of the charge lepton field. Planck scale effects will add other contribution to the mass matrix that gives the new mixing matrix can be written as $[20,21]$

$$
\begin{gathered}
U^{\prime}=U(1+i \delta \theta), \\
=\left(\begin{array}{cccc}
U_{s 1} & U_{s 2} & U_{s 3} & U_{s 4} \\
U_{e 1} & U_{e 2} & U_{e 3} & U_{e 4} \\
U_{\mu 1} & U_{\mu 2} & U_{\mu 3} & U_{\mu 4} \\
U_{\tau 1} & U_{\tau 2} & U_{\tau 3} & U_{\tau 4}
\end{array}\right) \\
+i\left(\begin{array}{cccc}
\sum_{i} U_{s i} \delta \theta_{i 1} & \sum_{i} U_{s i} \delta \theta_{i 2} & \sum_{i} U_{s i} \delta \theta_{i 3} & \sum_{i} U_{s i} \delta \theta_{i 4} \\
\sum_{i} U_{e i} \delta \theta_{i 1} & \sum_{i} U_{e i} \delta \theta_{i 2} & \sum_{i} U_{e i} \delta \theta_{i 3} & \sum_{i} U_{e i} \delta \theta_{i 4} \\
\sum_{i} U_{\mu i} \delta \theta_{i 1} & \sum_{i} U_{\mu i} \delta \theta_{i 2} & \sum_{i} U_{\mu i} \delta \theta_{i 3} & \sum_{i} U_{\mu i} \delta \theta_{i 4} \\
\sum_{i} U_{\tau i} \delta \theta_{i 1} & \sum_{i} U_{\tau i} \delta \theta_{i 2} & \sum_{i} U_{\tau i} \delta \theta_{i 3} & \sum_{i} U_{\tau i} \delta \theta_{i 4}
\end{array}\right)
\end{gathered}
$$

Where $\delta \theta$ is a hermition matrix that is first order in $\mu$ [20, 21]. The first order mass square difference $\Delta M_{i j}^{2}=M_{i}^{2}-M_{j}^{2}$, get modified [20,21] as

$$
\Delta M_{i j}^{\prime 2}=\Delta M_{i j}^{2}+2\left(M_{i} \operatorname{Re}\left(m_{i i}\right)-M_{j} \operatorname{Re}\left(m_{j j}\right)\right),
$$

and

$$
m=\mu U^{t} \lambda U
$$

where

$$
\mu=\frac{v^{2}}{M_{p l}}=2.5 \times 10^{-6} e V .
$$

The change in the elements of the mixing matrix, which we parametrized by $\delta \theta[20,21]$, is given by

$$
\delta \theta_{i j}=\frac{i \operatorname{Re}\left(m_{i j}\right)\left(M_{i}+M_{j}\right)-\operatorname{Im}\left(m_{i j}\right)\left(M_{i}-M_{j}\right)}{\Delta M_{i j}^{\prime 2}} .
$$

The above equation determine only the off diagonal elements of matrix $\delta \theta_{i j}$. The diagonal element of $\delta \theta_{i j}$ can be set to zero by phase invariance.

Using (19), we can calculate neutrino mixing angle due to Planck scale effects [31],

$$
\begin{gathered}
\sin ^{2} \theta_{14}^{\prime}=\left|\mathrm{U}_{\mathrm{s} 4}^{\prime}\right|^{2}, \\
\sin ^{2} \theta_{24}^{\prime}=\frac{\left|\mathrm{U}_{\mathrm{e} 4}^{\prime}\right|^{2}}{1-\left|\mathrm{U}_{\mathrm{s} 4}^{\prime}\right|^{2}},
\end{gathered}
$$




$$
\begin{gathered}
\sin ^{2} \theta_{34}^{\prime}=\frac{\left|\mathrm{U}_{\mu 4}^{\prime}\right|^{2}}{1-\left|\mathrm{U}_{\mathrm{s} 4}^{\prime}\right|^{2}-\left|\mathrm{U}_{\mathrm{s} 4}^{\prime}\right|^{2}}, \\
\sin ^{2} \theta_{13}^{\prime}=\frac{\left|\mathrm{U}_{\mathrm{s} 3}^{\prime}\right|^{2}}{1-\left|\mathrm{U}_{\mathrm{s} 4}^{\prime}\right|^{2}}, \\
\sin ^{2} \theta_{12}^{\prime}=\frac{\left|\mathrm{U}_{\mathrm{s} 2}^{\prime}\right|^{2}}{1-\left|\mathrm{U}_{\mathrm{s} 4}^{\prime}\right|^{2}-\left|\mathrm{U}_{\mathrm{s} 3}^{\prime}\right|^{2}}, \\
\sin ^{2} \theta_{23}^{\prime}=\frac{\left|\mathrm{U}_{\mathrm{e} 3}^{\prime}\right|^{2}\left(1-\left|\mathrm{U}_{\mathrm{s} 4}^{\prime}\right|^{2}\right)-\left(\left|\mathrm{U}_{\mathrm{s} 4}^{\prime}\right|^{2}\left|\mathrm{U}_{\mathrm{e} 4}^{\prime}\right|^{2}\right)}{1-\left|\mathrm{U}_{\mathrm{s} 4}^{\prime}\right|^{2}-\left|\mathrm{U}_{\mathrm{e} 4}^{\prime}\right|^{2}} \\
+\frac{\left|U_{s 1}^{\prime} U_{e 1}^{\prime}+U_{s 2}^{\prime} U_{e 2}^{\prime}\right|^{2}\left(1-\left|U_{s 4}^{\prime}\right|^{2}\right)}{\left(1-\left|U_{s 4}^{\prime}\right|^{2}-\left|U_{s 3}^{\prime}\right|^{2}\right)\left(1-\left|U_{s 4}^{\prime}\right|^{2}-\left|U_{e 4}^{\prime}\right|^{2}\right)}
\end{gathered}
$$

where,

$$
\begin{gathered}
U_{\alpha 1}^{\prime}=U_{\alpha 1}+\sum_{i} U_{\alpha i}\left(\frac{-\operatorname{Re}\left(m_{i 1}\right)\left(M_{i}+M_{1}\right)-i \operatorname{Im}\left(m_{i 1}\right)\left(M_{i}-M_{1}\right)}{M_{i}^{2}-M_{1}^{2}+2\left(M_{i} \operatorname{Re}\left(m_{i i}\right)-M_{1} \operatorname{Re}\left(m_{11}\right)\right.}\right), \\
U_{\alpha 2}^{\prime}=U_{\alpha 2}+\sum_{i} U_{\alpha i}\left(\frac{-\operatorname{Re}\left(m_{i 2}\right)\left(M_{i}+M_{2}\right)-i \operatorname{Im}\left(m_{i 2}\right)\left(M_{i}-M_{2}\right)}{M_{i}^{2}-M_{2}^{2}+2\left(M_{i} \operatorname{Re}\left(m_{i i}\right)-M_{4} \operatorname{Re}\left(m_{22}\right)\right.}\right), \\
U_{\alpha 3}^{\prime}=U_{\alpha 3}+\sum_{i} U_{\alpha i}\left(\frac{-\operatorname{Re}\left(m_{i 3}\right)\left(M_{i}+M_{3}\right)-i \operatorname{Im}\left(m_{i 3}\right)\left(M_{i}-M_{3}\right)}{M_{i}^{2}-M_{3}^{2}+2\left(M_{i} \operatorname{Re}\left(m_{i i}\right)-M_{4} \operatorname{Re}\left(m_{33}\right)\right.}\right), \\
\mathrm{U}_{\alpha 4}^{\prime}=\mathrm{U}_{\alpha 4}+\sum_{i} \mathrm{U}_{\alpha \mathrm{i}}\left(\frac{-\operatorname{Re}\left(\mathrm{m}_{\mathrm{i} 4}\right)\left(\mathrm{M}_{\mathrm{i}}+\mathrm{M}_{4}\right)-\mathrm{i} \operatorname{Im}\left(\mathrm{m}_{\mathrm{i} 4}\right)\left(\mathrm{M}_{\mathrm{i}}-\mathrm{M}_{4}\right)}{\mathrm{M}_{i}^{2}-\mathrm{M}_{4}^{2}+2\left(\mathrm{M}_{\mathrm{i}} \operatorname{Re}\left(\mathrm{m}_{\mathrm{ii}}\right)-\mathrm{M}_{4} \operatorname{Re}\left(\mathrm{m}_{44}\right)\right.} .\right), \quad \alpha=\mathrm{s}, \mathrm{e}, \mu, \tau
\end{gathered}
$$

\section{Jarlskog Above the GUT Scale}

Normal order or inverted order neutrino mass spectrum crucially depends on the neutrino mixing angles define in (23-28) and plank scale correction has very subtle effect on these spectrums. Thus, we consider a degenerate neutrino spectrum and take the common neutrino mass to $2 \mathrm{eV}$, which is the upper limit from the tritium decay experiment [25]. Let us 
compute new nine Jarlskog determiant due to new mixing due to Planck scale effects. which is simply given by replacing the matrix $U$ by perturbed matrix $U^{\prime}$,

$$
\begin{aligned}
& J_{s e}^{13^{\prime}}=\operatorname{Im}\left(\left(U_{s 1}+i \sum_{i} U_{s i} \delta \theta_{i 1}\right)\left(U_{e 3}+i \sum_{i} U_{e i} \delta \theta_{i 3}\right)\left(U_{s 3}^{*}-i \sum_{i} U_{e i}^{*} \delta \theta_{i 3}^{*}\right)\left(U_{e 1}^{*}-i \sum_{i} U_{e i}^{*} \delta \theta_{i 1}^{*}\right)\right) \\
& J_{s e}^{22^{\prime}}=\operatorname{Im}\left(\left(U_{s 2}+i \sum_{i} U_{s i} \delta \theta_{i 2}\right)\left(U_{e 4}+i \sum_{i} U_{e i} \delta \theta_{i 4}\right)\left(U_{s 4}^{*}-i \sum_{i} U_{e i}^{*} \delta \theta_{i 4}^{*}\right)\left(U_{e 2}^{*}-i \sum_{i} U_{e i}^{*} \delta \theta_{i 2}^{*}\right)\right) \\
& J_{s e}^{34^{\prime}}=\operatorname{Im}\left(\left(U_{s 3}+i \sum_{i} U_{s i} \delta \theta_{i 3}\right)\left(U_{e 4}+i \sum_{i} U_{e i} \delta \theta_{i 4}\right)\left(U_{s 4}^{*}-i \sum_{i} U_{e i}^{*} \delta \theta_{i 4}^{*}\right)\left(U_{e 3}^{*}-i \sum_{i} U_{e i}^{*} \delta \theta_{i 3}^{*}\right)\right) \\
& J_{\tau s}^{13^{\prime}}=\operatorname{Im}\left(\left(U_{\tau 1}+i \sum_{i} U_{\tau i} \delta \theta_{i 1}\right)\left(U_{s 3}+i \sum_{i} U_{e i} \delta \theta_{i 3}\right)\left(U_{\tau 3}^{*}-i \sum_{i} U_{\tau i}^{*} \delta \theta_{i 1}^{*}\right)\left(U_{s 1}^{*}-i \sum_{i} U_{s i}^{*} \delta \theta_{i 1}^{*}\right)\right) \\
& J_{\tau s}^{14^{\prime}}=\operatorname{Im}\left(\left(U_{\tau 1}+i \sum_{i} U_{\tau i} \delta \theta_{i 1}\right)\left(U_{s 4}+i \sum_{i} U_{e i} \delta \theta_{i 4}\right)\left(U_{\tau 4}^{*}-i \sum_{i} U_{\tau i}^{*} \delta \theta_{i 4}^{*}\right)\left(U_{s 1}^{*}-i \sum_{i} U_{s i}^{*} \delta \theta_{i 1}^{*}\right)\right) \\
& J_{\tau s}^{34^{\prime}}=\operatorname{Im}\left(\left(U_{\tau 3}+i \sum_{i} U_{\tau i} \delta \theta_{i 3}\right)\left(U_{s 4}+i \sum_{i} U_{e i} \delta \theta_{i 4}\right)\left(U_{\tau 4}^{*}-i \sum_{i} U_{\tau i}^{*} \delta \theta_{i 4}^{*}\right)\left(U_{s 3}^{*}-i \sum_{i} U_{s i}^{*} \delta \theta_{i 3}^{*}\right)\right) \\
& J_{e \mu}^{23^{\prime}}=\operatorname{Im}\left(\left(U_{e 2}+i \sum_{i} U_{e i} \delta \theta_{i 2}\right)\left(U_{\mu 3}+i \sum_{i} U_{\mu i} \delta \theta_{i 3}\right)\left(U_{e 3}^{*}-i \sum_{i} U_{e i}^{*} \delta \theta_{i 3}^{*}\right)\left(U_{\mu 2}^{*}-i \sum_{i} U_{\mu i}^{*} \delta \theta_{i 2}^{*}\right)\right) \\
& J_{e \mu}^{24^{\prime}}=\operatorname{Im}\left(\left(U_{e 2}+i \sum_{i} U_{e i} \delta \theta_{i 2}\right)\left(U_{\mu 4}+i \sum_{i} U_{\mu i} \delta \theta_{i 4}\right)\left(U_{e 4}^{*}-i \sum_{i} U_{e i}^{*} \delta \theta_{i 4}^{*}\right)\left(U_{\mu 2}^{*}-i \sum_{i} U_{\mu i}^{*} \delta \theta_{i 2}^{*}\right)\right) \\
& J_{e \mu}^{34^{\prime}}=\operatorname{Im}\left(\left(U_{e 3}+i \sum_{i} U_{e i} \delta \theta_{i 3}\right)\left(U_{\mu 4}+i \sum_{i} U_{\mu i} \delta \theta_{i 4}\right)\left(U_{e 4}^{*}-i \sum_{i} U_{e i}^{*} \delta \theta_{i 4}^{*}\right)\left(U_{\mu 3}^{*}-i \sum_{i} U_{\mu i}^{*} \delta \theta_{i 3}^{*}\right)\right)
\end{aligned}
$$

In term of mixing angle and Dirac phases, we can write Jarlskog determinant $J_{\alpha \beta}^{i j^{\prime}}$ above the GUT scale are,

$$
\begin{aligned}
& J_{s e}^{13^{\prime}}=\frac{1}{16} \sin 2 \theta_{12}^{\prime} \sin 2 \theta_{13}^{\prime} \sin 2 \theta_{14}^{\prime} \sin 2 \theta_{24}^{\prime} \cos \theta_{14}^{\prime} \cos \theta_{23}^{\prime} \sin \theta_{13}^{\prime} \sin \theta_{y} \\
& -\frac{1}{16} \sin 2 \theta_{12}^{\prime} \sin 2 \theta_{13}^{\prime} \sin 2 \theta_{14}^{\prime} \sin 2 \theta_{23}^{\prime} \cos \theta_{14}^{\prime} \cos ^{2} \theta_{24}^{\prime} \sin \theta_{z} \\
& +\frac{1}{8} \sin 2 \theta_{13}^{\prime} \sin 2 \theta_{14}^{\prime} \sin 2 \theta_{24}^{\prime} \cos ^{2} \theta_{12}^{\prime} \cos \theta_{14}^{\prime} \sin \theta_{23}^{\prime} \sin \left(\theta_{y}-\theta_{z}\right) \\
& J_{s e}^{24^{\prime}}=\frac{1}{8} \sin 2 \theta_{12}^{\prime} \sin 2 \theta_{14}^{\prime} \sin 2 \theta_{24}^{\prime} \cos \theta_{13}^{\prime} \cos \theta_{14}^{\prime} \cos \theta_{23}^{\prime} \sin \theta_{y} \\
& +\frac{1}{4} \sin 2 \theta_{13}^{\prime} \sin 2 \theta_{14}^{\prime} \sin ^{2} \theta_{12}^{\prime} \cos \theta_{14}^{\prime} \sin \theta_{24}^{\prime} \sin \theta_{23}^{\prime} \sin \left(\theta_{y}-\theta_{z}\right) \\
& J_{s e}^{34^{\prime}}=\frac{1}{8} \sin 2 \theta_{13}^{\prime} \sin 2 \theta_{14}^{\prime} \sin 2 \theta_{24}^{\prime} \cos \theta_{14}^{\prime} \sin \theta_{23}^{\prime} \sin \left(\theta_{y}-\theta_{z}\right) \\
& J_{\tau s}^{13^{\prime}}=\frac{1}{8} \sin 2 \theta_{13}^{\prime} \sin 2 \theta_{14}^{\prime} \sin 2 \theta_{34}^{\prime} \cos ^{2} \theta_{12}^{\prime} \cos \theta_{14}^{\prime} \cos \theta_{23}^{\prime} \cos \theta_{24}^{\prime} \sin \theta_{x} \\
& +\frac{1}{16} \sin 2 \theta_{12}^{\prime} \sin 2 \theta_{13}^{\prime} \sin 2 \theta_{14}^{\prime} \sin 2 \theta_{24}^{\prime} \cos \theta_{14}^{\prime} \cos \theta_{23}^{\prime} \cos ^{2} \theta_{34}^{\prime} \sin \theta_{y} \\
& +\frac{1}{8}\left(\cos ^{2} \theta_{34}^{\prime} \sin ^{2} \theta_{24}^{\prime}-\sin ^{2} \theta_{34}^{\prime}\right) \sin 2 \theta_{12}^{\prime} \sin 2 \theta_{13}^{\prime} \sin 2 \theta_{23}^{\prime} \cos \theta_{13}^{\prime} \cos ^{2} \theta_{14}^{\prime} \sin \theta_{z} \\
& -\frac{1}{8} \sin 2 \theta_{12}^{\prime} \sin 2 \theta_{13}^{\prime} \sin 2 \theta_{34}^{\prime} \cos \theta_{13}^{\prime} \cos ^{2} \theta_{14}^{\prime} \cos ^{2} \theta_{23}^{\prime} \sin \theta_{24}^{\prime} \sin \left(\theta_{x}-\theta_{y}\right) \\
& -\frac{1}{16} \sin 2 \theta_{12}^{\prime} \sin 2 \theta_{13}^{\prime} \sin 2 \theta_{14}^{\prime} \sin 2 \theta_{34}^{\prime} \cos \theta_{14}^{\prime} \cos \theta_{24}^{\prime} \sin \theta_{13}^{\prime} \sin \theta_{23}^{\prime} \sin \left(\theta_{x}+\theta_{z}\right) \\
& +\frac{1}{8} \sin 2 \theta_{13}^{\prime} \sin 2 \theta_{14}^{\prime} \sin 2 \theta_{24}^{\prime} \cos ^{2} \theta_{12}^{\prime} \cos \theta_{14}^{\prime} \cos ^{2} \theta_{34}^{\prime} \sin \theta_{23}^{\prime} \sin \left(\theta_{y}-\theta_{z}\right) \\
& -\frac{1}{8} \sin 2 \theta_{12}^{\prime} \sin 2 \theta_{13}^{\prime} \sin 2 \theta_{34}^{\prime} \cos ^{2} \theta_{14}^{\prime} \cos \theta_{13}^{\prime} \sin ^{2} \theta_{23}^{\prime} \sin \theta_{24}^{\prime} \sin \left(\theta_{x}-\theta_{y}+\theta_{z}\right)
\end{aligned}
$$




$$
\begin{aligned}
J_{\tau s}^{14^{\prime}}= & -\frac{1}{8} \sin 2 \theta_{13}^{\prime} \sin 2 \theta_{14}^{\prime} \sin 2 \theta_{34}^{\prime} \cos ^{2} \theta_{12}^{\prime} \cos \theta_{14}^{\prime} \cos \theta_{23}^{\prime} \cos \theta_{24}^{\prime} \sin \theta_{x} \\
& -\frac{1}{8} \sin 2 \theta_{12}^{\prime} \sin 2 \theta_{13}^{\prime} \sin 2 \theta_{24}^{\prime} \cos ^{2} \theta_{14}^{\prime} \cos \theta_{23}^{\prime} \cos ^{2} \theta_{34}^{\prime} \sin \theta_{y} \\
& -\frac{1}{8} \sin 2 \theta_{12}^{\prime} \sin 2 \theta_{14}^{\prime} \sin 2 \theta_{34}^{\prime} \cos \theta_{13}^{\prime} \cos \theta_{14}^{\prime} \cos \theta_{24}^{\prime} \sin \theta_{23}^{\prime} \sin \left(\theta_{x}+\theta_{z}\right) \\
& -\frac{1}{8} \sin 2 \theta_{12}^{\prime} \sin 2 \theta_{13}^{\prime} \sin 2 \theta_{24}^{\prime} \cos ^{2} \theta_{14}^{\prime} \cos \theta_{12}^{\prime} \cos ^{2} \theta_{34}^{\prime} \sin \theta_{23}^{\prime} \sin \left(\theta_{y}-\theta_{z}\right)
\end{aligned}
$$

$$
\begin{aligned}
J_{\tau s}^{34^{\prime}}= & \frac{1}{8} \sin 2 \theta_{13}^{\prime} \sin 2 \theta_{14}^{\prime} \sin 2 \theta_{34}^{\prime} \cos \theta_{14}^{\prime} \cos \theta_{23}^{\prime} \cos \theta_{24}^{\prime} \sin \theta_{x} \\
& +\frac{1}{8} \sin 2 \theta_{13}^{\prime} \sin 2 \theta_{14}^{\prime} \sin 2 \theta_{24}^{\prime} \cos \theta_{14}^{\prime} \cos ^{2} \theta_{34}^{\prime} \sin \theta_{23}^{\prime} \sin \left(\theta_{y}-\theta_{z}\right)
\end{aligned}
$$

$$
\begin{aligned}
& J_{e \mu}^{23^{\prime}}=-\frac{1}{4}\left(\cos ^{2} \theta_{12}^{\prime} \cos ^{2} \theta_{23}^{\prime} \sin ^{2} \theta_{24}^{\prime}-\cos ^{2} \theta_{12}^{\prime} \cos ^{2} \theta_{24}^{\prime} \sin ^{2} \theta_{23}^{\prime}+\sin ^{2} \theta_{12}^{\prime} \sin ^{2} \theta_{14}^{\prime} \sin ^{2} \theta_{24}^{\prime}\right. \\
& \left.+\sin ^{2} \theta_{12}^{\prime} \sin ^{2} \theta_{23}^{\prime}\right) \sin 2 \theta_{13}^{\prime} \sin 2 \theta_{34}^{\prime} \sin \theta_{14}^{\prime} \cos \theta_{23}^{\prime} \cos \theta_{24}^{\prime} \sin \theta_{x} \\
& +\frac{1}{4}\left(\cos ^{2} \theta_{13}^{\prime} \cos ^{2} \theta_{34}^{\prime} \sin ^{2} \theta_{23}^{\prime}-\cos ^{2} \theta_{23}^{\prime} \cos ^{2} \theta_{34}^{\prime} \sin ^{2} \theta_{13}^{\prime}+\sin ^{2} \theta_{13}^{\prime} \sin ^{2} \theta_{14}^{\prime} \sin ^{2} \theta_{34}^{\prime}\right. \\
& \left.-\sin ^{2} \theta_{34}^{\prime} \sin ^{2} \theta_{23}^{\prime}\right) \sin 2 \theta_{12}^{\prime} \sin 2 \theta_{24}^{\prime} \sin \theta_{14}^{\prime} \cos \theta_{13}^{\prime} \cos \theta_{23}^{\prime} \cos \theta_{24}^{\prime} \sin \theta_{y} \\
& +\frac{1}{8}\left(\cos ^{2} \theta_{24}^{\prime} \cos ^{2} \theta_{34}^{\prime}-\cos ^{2} \theta_{24}^{\prime} \sin ^{2} \theta_{14}^{\prime} \sin ^{2} \theta_{34}^{\prime}-\cos ^{2} \theta_{34}^{\prime} \sin ^{2} \theta_{14}^{\prime} \sin ^{2} \theta_{24}^{\prime}\right. \\
& \left.+\sin ^{2} \theta_{14}^{\prime} \sin ^{2} \theta_{24}^{\prime} \sin ^{2} \theta_{34}^{\prime}\right) \sin 2 \theta_{12}^{\prime} \sin 2 \theta_{13}^{\prime} \sin 2 \theta_{23}^{\prime} \cos \theta_{13}^{\prime} \cos \theta_{34}^{\prime} \sin \theta_{z} \\
& +\frac{1}{4}\left(\sin ^{2} \theta_{34}^{\prime} \cos ^{2} \theta_{13}^{\prime}-\cos ^{2} \theta_{24}^{\prime} \sin ^{2} \theta_{13}^{\prime}\right) \sin 2 \theta_{12}^{\prime} \sin 2 \theta_{34}^{\prime} \\
& \cos ^{2} \theta_{23}^{\prime} \sin \theta_{13}^{\prime} \sin ^{2} \theta_{14}^{\prime} \sin \theta_{24}^{\prime} \sin \left(\theta_{x}-\theta_{y}\right) \\
& +\frac{1}{4}\left(\cos ^{2} \theta_{23}^{\prime}-\sin ^{2} \theta_{24}^{\prime} \cos ^{2} \theta_{13}^{\prime}-\cos ^{2} \theta_{24}^{\prime} \sin ^{2} \theta_{13}^{\prime}+\sin ^{2} \theta_{13}^{\prime} \sin ^{2} \theta_{14}^{\prime} \sin ^{2} \theta_{24}^{\prime}\right) \\
& \sin 2 \theta_{12}^{\prime} \sin 2 \theta_{34}^{\prime} \cos \theta_{13}^{\prime} \cos \theta_{24}^{\prime} \sin \theta_{23}^{\prime} \sin \left(\theta_{x}+\theta_{z}\right) \\
& -\frac{1}{4}\left(\cos ^{2} \theta_{12}^{\prime} \cos ^{2} \theta_{23}^{\prime} \cos ^{2} \theta_{34}^{\prime}-\cos ^{2} \theta_{12}^{\prime} \cos ^{2} \theta_{23}^{\prime} \sin ^{2} \theta_{34}^{\prime}-\cos ^{2} \theta_{23}^{\prime} \cos ^{2} \theta_{34}^{\prime} \sin ^{2} \theta_{12}^{\prime}\right. \\
& \left.+\sin ^{2} \theta_{12}^{\prime} \sin ^{2} \theta_{14}^{\prime} \sin ^{2} \theta_{34}^{\prime}-\sin ^{2} \theta_{12}^{\prime} \sin ^{2} \theta_{23}^{\prime} \sin ^{2} \theta_{34}^{\prime}\right) \sin 2 \theta_{13}^{\prime} \sin 2 \theta_{24}^{\prime} \sin \theta_{14}^{\prime} \sin \theta_{23}^{\prime} \sin \left(\theta_{y}-\theta_{z}\right) \\
& +\frac{1}{4}\left(\cos ^{2} \theta_{12}^{\prime} \cos ^{2} \theta_{13}^{\prime} \cos ^{2} \theta_{24}^{\prime}-\cos ^{2} \theta_{12}^{\prime} \cos ^{2} \theta_{24}^{\prime} \sin ^{2} \theta_{13}^{\prime} \sin ^{2} \theta_{14}^{\prime}-\cos ^{2} \theta_{13}^{\prime} \cos ^{2} \theta_{24}^{\prime} \sin ^{2} \theta_{12}^{\prime} \sin ^{2} \theta_{14}^{\prime}\right. \\
& \left.+\cos ^{2} \theta_{24}^{\prime} \sin ^{2} \theta_{12}^{\prime} \sin ^{2} \theta_{13}^{\prime} \sin ^{2} \theta_{14}^{\prime}\right) \sin 2 \theta_{23}^{\prime} \sin 2 \theta_{34}^{\prime} \sin \theta_{23}^{\prime} \sin \left(\theta_{x}-\theta_{y}+\theta_{z}\right) \\
& -\frac{1}{4}\left(\cos ^{2} \theta_{13}^{\prime} \cos ^{2} \theta_{24}^{\prime} \sin ^{2} \theta_{23}^{\prime}-\cos ^{2} \theta_{13}^{\prime} \sin ^{2} \theta_{23}^{\prime} \sin ^{2} \theta_{24}^{\prime} \sin ^{2} \theta_{14}^{\prime}-\cos ^{2} \theta_{24}^{\prime} \sin ^{2} \theta_{23}^{\prime} \sin ^{2} \theta_{13}^{\prime} \sin ^{2} \theta_{14}^{\prime}\right) \\
& \sin 2 \theta_{12}^{\prime} \sin 2 \theta_{34}^{\prime} \sin \theta_{13}^{\prime} \sin \theta_{24}^{\prime} \sin \left(\theta_{x}-\theta_{y}+2 \theta_{z}\right) \\
& -\frac{1}{16} \sin 2 \theta_{12}^{\prime} \sin 2 \theta_{13}^{\prime} \sin 2 \theta_{24}^{\prime} \sin 2 \theta_{34}^{\prime} \cos \theta_{13}^{\prime} \cos \theta_{24}^{\prime} \sin ^{2} \theta_{14}^{\prime} \sin \left(\theta_{x}+\theta_{y}\right) \\
& +\frac{1}{16} \sin 2 \theta_{12}^{\prime} \sin 2 \theta_{13}^{\prime} \sin 2 \theta_{23}^{\prime} \sin 2 \theta_{34}^{\prime} \sin \theta_{13}^{\prime} \cos \theta_{24}^{\prime} \sin \theta_{14}^{\prime} \sin \left(\theta_{x}-\theta_{z}\right) \\
& +\frac{1}{8}\left(\cos ^{2} \theta_{34}^{\prime}-\sin ^{2} \theta_{34}^{\prime}\right) \sin 2 \theta_{24}^{\prime} \sin 2 \theta_{23}^{\prime} \sin 2 \theta_{34}^{\prime} \sin \theta_{13}^{\prime} \cos \theta_{12}^{\prime} \sin \theta_{24}^{\prime} \sin \theta_{14}^{\prime} \sin \theta_{23}^{\prime} \sin \left(\theta_{y}-2 \theta_{z}\right) \\
& -\frac{1}{8}\left(\cos ^{2} \theta_{12}^{\prime}-\sin ^{2} \theta_{12}^{\prime}\right) \sin 2 \theta_{13}^{\prime} \sin 2 \theta_{23}^{\prime} \sin 2 \theta_{34}^{\prime} \sin ^{2} \theta_{24}^{\prime} \cos \theta_{24}^{\prime} \sin \theta_{14}^{\prime} \sin \theta_{23}^{\prime} \sin \left(\theta_{x}-2 \theta_{y}+2 \theta_{z}\right) \\
& -\frac{1}{8} \sin 2 \theta_{12}^{\prime} \sin 2 \theta_{23}^{\prime} \sin 2 \theta_{34}^{\prime} \cos \theta_{12}^{\prime} \cos \theta_{23}^{\prime} \sin \theta_{24}^{\prime} \cos \theta_{34}^{\prime} \sin \theta_{14}^{\prime} \sin \theta_{34}^{\prime} \sin \left(\theta_{x}-2 \theta_{y}+\theta_{z}\right) \\
& +\frac{1}{16} \sin 2 \theta_{12}^{\prime} \sin 2 \theta_{13}^{\prime} \sin 2 \theta_{24}^{\prime} \sin 2 \theta_{34}^{\prime} \sin \theta_{13}^{\prime} \sin \theta_{14}^{\prime} \sin ^{2} \theta_{23}^{\prime} \sin \theta_{24}^{\prime} \sin \left(\theta_{x}-2 \theta_{y}+3 \theta_{z}\right)
\end{aligned}
$$


Table 1 Some value of Jarlskog Determinant above GUT scale for various value of Majorana phases. Input value of mixing angles $\theta_{13}=10^{\circ}, \theta_{23}=45^{\circ}, \theta_{12}=34^{\circ}, \theta_{34}=18.5^{\circ}, \theta_{24}=4^{o}, \theta_{14}=3.6^{o}$

\begin{tabular}{|c|c|c|c|c|c|}
\hline$\alpha$ & $\beta$ & $\gamma$ & $J_{s e}^{\prime} \times 10^{-4}$ & $J_{e \mu}^{\prime} \times 10^{-4}$ & $J_{\tau s}^{\prime} \times 10^{-4}$ \\
\hline $0^{0}$ & $45^{0}$ & $0^{0}$ & 16.3268 & -16.3216 & 0.1575 \\
\hline $0^{0}$ & $45^{0}$ & $45^{0}$ & 11.1673 & -12.0438 & -0.7514 \\
\hline $0^{0}$ & $45^{0}$ & $90^{0}$ & 15.9772 & -16.226 & 0.1293 \\
\hline $0^{0}$ & $45^{0}$ & $135^{0}$ & 21.1395 & -20.5047 & 1.0385 \\
\hline $0^{0}$ & $45^{0}$ & $180^{\circ}$ & 16.3268 & -16.3216 & 0.1575 \\
\hline $0^{0}$ & $135^{0}$ & $0^{0}$ & -16.3268 & 16.3216 & -0.1575 \\
\hline $0^{0}$ & $135^{0}$ & $90^{0}$ & -15.9772 & 16.226 & -0.1293 \\
\hline $0^{0}$ & $135^{0}$ & $180^{\circ}$ & -16.3268 & 16.3216 & -0.1575 \\
\hline $45^{0}$ & $45^{0}$ & $0^{0}$ & 4.9008 & -4.2205 & 0.8779 \\
\hline $45^{0}$ & $45^{0}$ & $45^{0}$ & -0.0007 & -0.0056 & -0.0036 \\
\hline $45^{0}$ & $45^{0}$ & $90^{0}$ & 4.9861 & -4.2947 & 0.8925 \\
\hline $45^{0}$ & $45^{0}$ & $135^{0}$ & 9.8869 & -8.5091 & 1.7739 \\
\hline $45^{0}$ & $45^{0}$ & $180^{\circ}$ & 4.9008 & -4.2205 & 0.8779 \\
\hline $45^{0}$ & $90^{0}$ & $0^{0}$ & -11.4116 & 12.1015 & 0.7409 \\
\hline $45^{0}$ & $90^{0}$ & $90^{\circ}$ & -10.9707 & 11.928 & 0.7845 \\
\hline $45^{0}$ & $90^{\circ}$ & $180^{\circ}$ & -11.4116 & 12.1015 & 0.7409 \\
\hline $45^{0}$ & $135^{0}$ & $0^{0}$ & -26.7413 & 27.5152 & 0.5718 \\
\hline $45^{0}$ & $135^{0}$ & $45^{0}$ & -31.3015 & 31.6378 & -0.2819 \\
\hline $45^{0}$ & $135^{0}$ & $90^{0}$ & -25.9765 & 27.2563 & 0.6412 \\
\hline $45^{0}$ & $135^{0}$ & $135^{0}$ & -21.4225 & 23.1361 & 1.4944 \\
\hline $45^{0}$ & $135^{0}$ & $180^{\circ}$ & -26.7413 & 27.5152 & 0.5718 \\
\hline $45^{0}$ & $180^{\circ}$ & $0^{0}$ & -10.4681 & 11.2299 & 0.7096 \\
\hline $45^{0}$ & $180^{\circ}$ & $90^{0}$ & -10.0577 & 11.0699 & 0.7503 \\
\hline $45^{0}$ & $180^{\circ}$ & $180^{\circ}$ & -10.4681 & 11.2299 & 0.7096 \\
\hline $90^{\circ}$ & $45^{0}$ & $0^{0}$ & 15.341 & -15.4407 & 0.1486 \\
\hline $90^{0}$ & $45^{0}$ & $90^{0}$ & 15.0103 & -15.3514 & 0.1219 \\
\hline $90^{0}$ & $45^{0}$ & $180^{\circ}$ & 15.341 & -15.4407 & 0.1486 \\
\hline $90^{0}$ & $135^{0}$ & $0^{0}$ & -15.341 & 15.4407 & -0.1486 \\
\hline $90^{\circ}$ & $135^{0}$ & $90^{0}$ & -15.0103 & 15.3514 & -0.1219 \\
\hline $90^{0}$ & $135^{0}$ & $180^{\circ}$ & -15.341 & 15.4407 & -0.1486 \\
\hline $135^{\circ}$ & $45^{0}$ & $0^{0}$ & 26.7413 & -27.5152 & -0.5718 \\
\hline $135^{0}$ & $45^{0}$ & $45^{0}$ & 21.4225 & -23.1361 & -1.4944 \\
\hline $135^{0}$ & $45^{0}$ & $90^{0}$ & 25.9765 & -27.2563 & -0.6412 \\
\hline $135^{0}$ & $45^{0}$ & $135^{0}$ & 31.3015 & -31.6378 & -0.2819 \\
\hline $135^{0}$ & $45^{0}$ & $180^{\circ}$ & 26.7413 & -27.5152 & -0.5718 \\
\hline $135^{0}$ & $90^{0}$ & $0^{0}$ & 11.4116 & -12.1015 & -0.7409 \\
\hline $135^{0}$ & $90^{\circ}$ & $90^{\circ}$ & 10.9707 & -11.928 & -0.7845 \\
\hline $135^{0}$ & $90^{\circ}$ & $180^{\circ}$ & 11.4116 & -12.1015 & -0.7409 \\
\hline $135^{0}$ & $135^{\circ}$ & $0^{0}$ & -4.9008 & 4.2205 & -0.8779 \\
\hline $135^{0}$ & $135^{0}$ & $45^{0}$ & -9.8868 & 8.5091 & -1.7739 \\
\hline $135^{0}$ & $135^{0}$ & $90^{\circ}$ & -4.9861 & 4.2947 & -0.8925 \\
\hline
\end{tabular}


Table 1 (continued)

\begin{tabular}{llllll}
\hline$\alpha$ & $\beta$ & $\gamma$ & $J_{s e}^{\prime} \times 10^{-4}$ & $J_{e \mu}^{\prime} \times 10^{-4}$ & $J_{\tau s}^{\prime} \times 10^{-4}$ \\
\hline $135^{0}$ & $135^{0}$ & $135^{0}$ & 0.0007 & 0.0056 & 0.0036 \\
$135^{0}$ & $135^{0}$ & $180^{0}$ & -4.9008 & 4.2205 & -0.8779 \\
$135^{0}$ & $180^{0}$ & $0^{0}$ & 10.4681 & -11.2299 & -0.7096 \\
$135^{0}$ & $180^{0}$ & $90^{0}$ & 10.0577 & -11.699 & -0.7503 \\
$135^{0}$ & $180^{0}$ & $180^{0}$ & 10.4681 & -11.2299 & -0.7096 \\
\hline
\end{tabular}

$$
\begin{aligned}
J_{e \mu}^{24^{\prime}}= & \frac{1}{16} \sin 2 \theta_{13}^{\prime} \sin 2 \theta_{14}^{\prime} \sin 2 \theta_{24}^{\prime} \sin 2 \theta_{34}^{\prime} \cos \theta_{14}^{\prime} \cos \theta_{23}^{\prime} \sin \theta_{14}^{\prime} \sin \theta_{24}^{\prime} \sin \theta_{x} \\
& +\frac{1}{8} \sin 2 \theta_{12}^{\prime} \sin 2 \theta_{14}^{\prime} \sin 2 \theta_{24}^{\prime} \cos \theta_{13}^{\prime} \cos \theta_{14}^{\prime} \cos \theta_{23}^{\prime} \sin ^{2} \theta_{34}^{\prime} \sin \theta_{x} \\
& -\frac{1}{4} \sin 2 \theta_{12}^{\prime} \sin 2 \theta_{24}^{\prime} \cos ^{2} \theta_{14}^{\prime} \cos ^{2} \theta_{23}^{\prime} \cos \theta_{24}^{\prime} \cos \theta_{34}^{\prime} \sin \theta_{13}^{\prime} \sin \theta_{34}^{\prime} \sin \left(\theta_{x}-\theta_{y}\right) \\
& +\frac{1}{16} \sin 2 \theta_{12}^{\prime} \sin 2 \theta_{14}^{\prime} \sin 2 \theta_{24}^{\prime} \sin 2 \theta_{34}^{\prime} \cos \theta_{13}^{\prime} \sin \theta_{23}^{\prime} \sin \theta_{24}^{\prime} \sin \left(\theta_{x}+\theta_{y}\right) \\
& -\frac{1}{8} \sin 2 \theta_{13}^{\prime} \sin 2 \theta_{14}^{\prime} \sin 2 \theta_{24}^{\prime} \cos \theta_{14}^{\prime} \sin ^{2} \theta_{12}^{\prime} \sin \theta_{23}^{\prime} \sin ^{2} \theta_{34}^{\prime} \sin \left(\theta_{y}-\theta_{z}\right) \\
& +\frac{1}{8} \sin 2 \theta_{12}^{\prime} \sin 2 \theta_{13}^{\prime} \sin 2 \theta_{34}^{\prime} \cos \theta_{13}^{\prime} \cos { }^{2} \theta_{14}^{\prime} \sin { }^{2} \theta_{23}^{\prime} \sin \theta_{24}^{\prime} \sin \left(\theta_{x}-\theta_{y}+2 \theta_{z}\right) \\
& -\frac{1}{4}\left(\cos { }^{2} \theta_{12}^{\prime}-\sin { }^{2} \theta_{12}^{\prime} \sin ^{2} \theta_{13}^{\prime}\right) \sin _{23}^{\prime} \sin 2 \theta_{24}^{\prime} \cos { }^{2} \theta_{14}^{\prime} \cos \theta_{34}^{\prime} \cos \theta_{24}^{\prime} \sin \theta_{34}^{\prime} \sin \left(\theta_{x}-\theta_{y}+\theta_{z}\right) \\
J_{e \mu}^{34^{\prime}}= & -\frac{1}{16} \sin 2 \theta_{13}^{\prime} \sin 2 \theta_{14}^{\prime} \sin 2 \theta_{24}^{\prime} \sin 2 \theta_{34}^{\prime} \cos \theta_{14}^{\prime} \cos \theta_{23}^{\prime} \sin \theta_{24}^{\prime} \sin \theta_{x} \\
& +\frac{1}{8} \sin 2 \theta_{13}^{\prime} \sin 2 \theta_{14}^{\prime} \sin 2 \theta_{24}^{\prime} \cos \theta_{14}^{\prime} \sin \theta_{23}^{\prime} \sin { }^{2} \theta_{34}^{\prime} \sin \left(\theta_{y}-\theta_{z}\right) \\
& +\frac{1}{4} \sin 2 \theta_{23}^{\prime} \sin 2 \theta_{24}^{\prime} \cos { }^{2} \theta_{13}^{\prime} \cos { }^{2} \theta_{14}^{\prime} \cos \theta_{24}^{\prime} \cos \theta_{34}^{\prime} \sin \theta_{34}^{\prime} \sin \left(\theta_{x}-\theta_{y}+\theta_{z}\right)
\end{aligned}
$$

where,

$$
\begin{aligned}
& \theta_{x}=\delta_{14}-\delta_{13}-\delta_{34} \\
& \theta_{y}=\delta_{14}-\delta_{12}-\delta_{24} \\
& \theta_{z}=\delta_{13}-\delta_{12}-\delta_{23}
\end{aligned}
$$

The values of six CP-violating phases $\delta_{i j}$ are between 0 and $2 \pi$.

\section{Numerical Results}

Since, the role of Jarkslog invariant that is apear in the oscillation probability (see (2)) seems to be in additive manner as given in (9). Hence, we are numerically calculated the collective role of Jarlskog invariant of a particular oscillation is given by

$$
\begin{aligned}
& J_{s e}^{\prime}=J_{s e}^{13^{\prime}}+J_{s e}^{24^{\prime}}+J_{s e}^{34^{\prime}} \\
& J_{\tau s}^{\prime}=J_{\tau s}^{13^{\prime}}+J_{\tau s}^{14^{\prime}}+J_{\tau s}^{34^{\prime}} \\
& J_{e \mu}^{\prime}=J_{e \mu}^{23^{\prime}}+J_{e \mu}^{24^{\prime}}+J_{e \mu}^{34^{\prime}}
\end{aligned}
$$




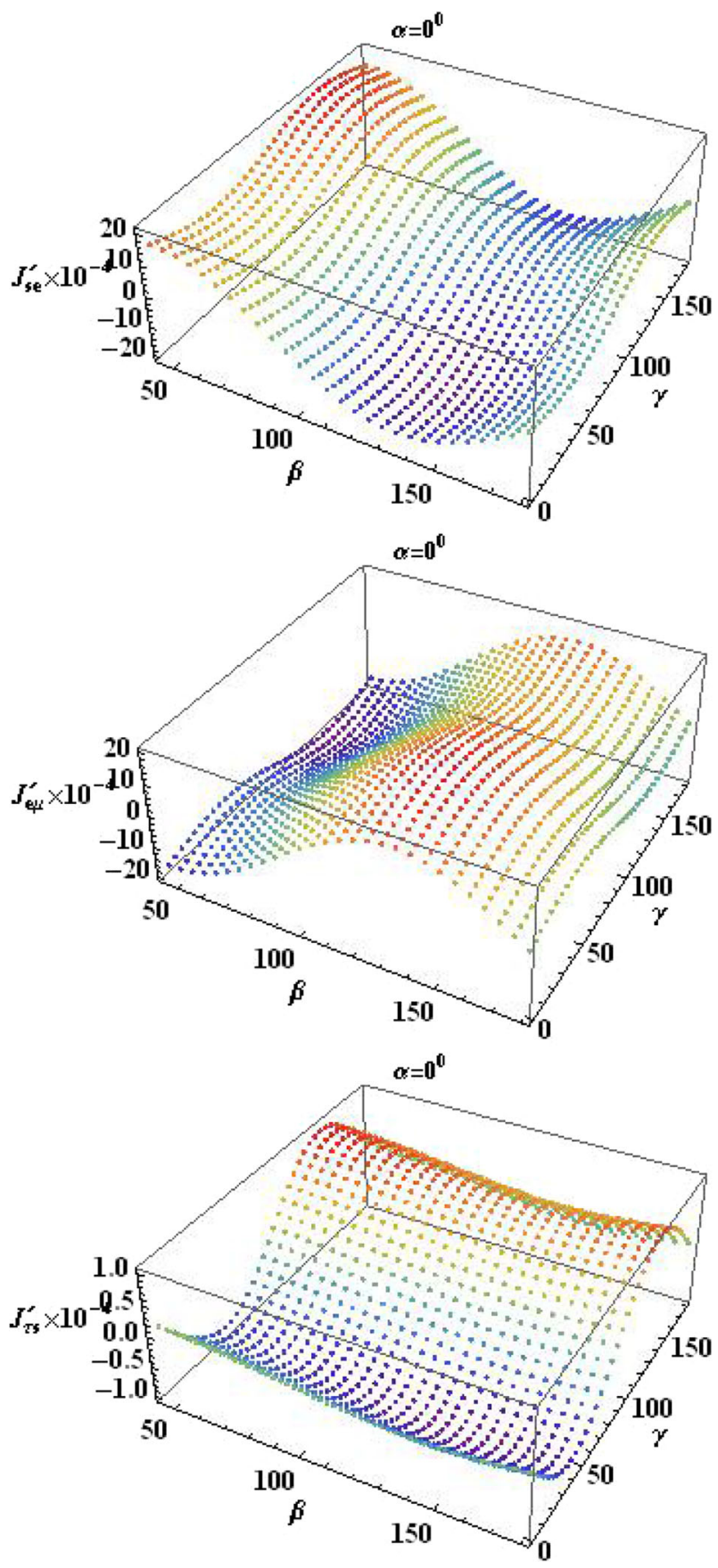

Fig. 1 For $\alpha=0^{0}$, the variation of $J_{s e}^{\prime}, J_{\tau s}^{\prime}$ and $J_{e \mu}^{\prime}$ with $\beta$ and $\gamma$. Input values are $\Delta_{31}=2.0 \times 10^{-3} e V^{2}$, $\Delta_{21}=8.0 \times 10^{-5} \mathrm{eV}^{2}, \Delta_{41}=1.7 \mathrm{eV}^{2}$ and mixing angles $\theta_{13}=10^{\circ}, \theta_{23}=45^{\circ}, \theta_{12}=34^{\circ}, \theta_{34}=$ $18.5^{\circ}, \theta_{24}=4^{o}, \theta_{14}=3.6^{\circ}$ 

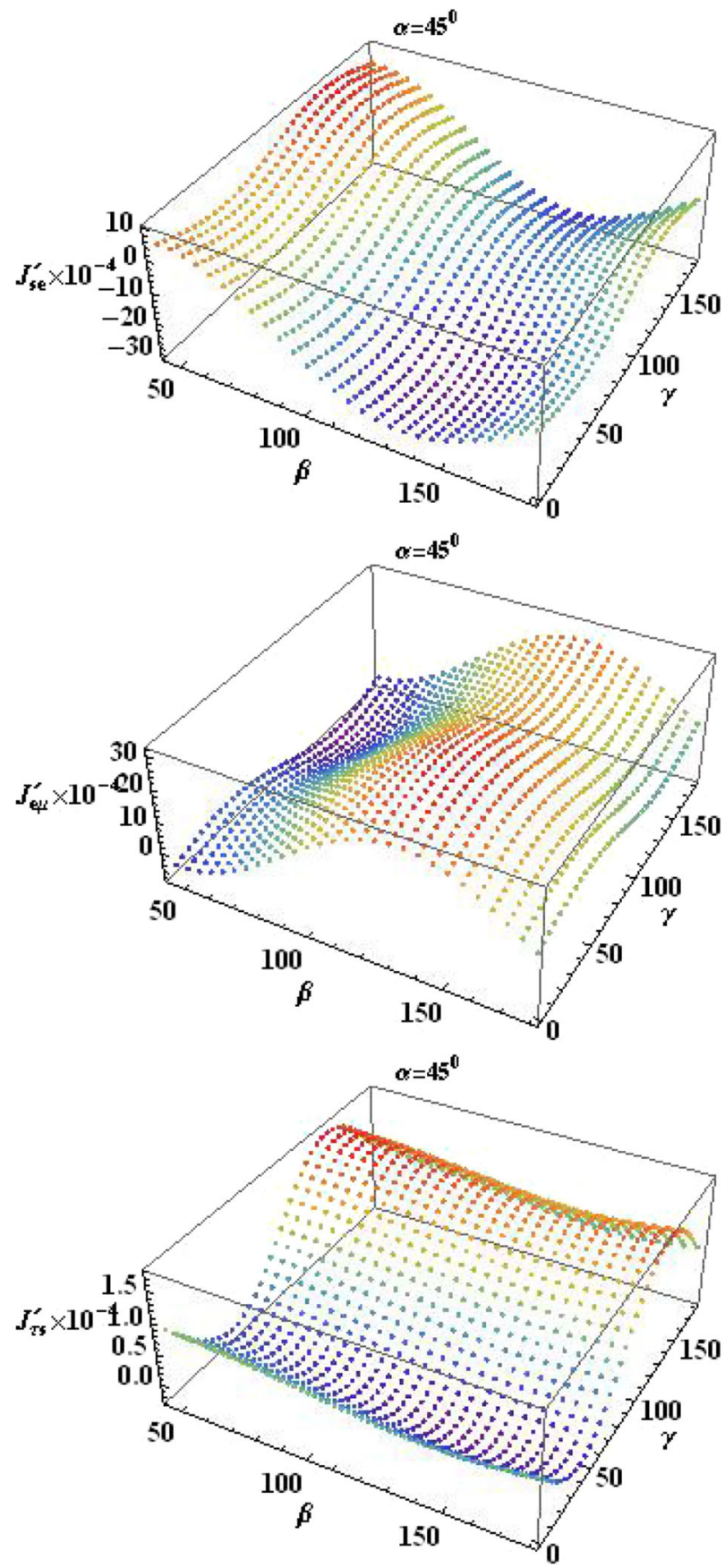

Fig. 2 For $\alpha=45^{\circ}$, the variation of $J_{s e}^{\prime}, J_{\tau s}^{\prime}$ and $J_{e \mu}^{\prime}$ with $\beta$ and $\gamma$. Input values are $\Delta_{31}=2.0 \times 10^{-3} e V^{2}$, $\Delta_{21}=8.0 \times 10^{-5} \mathrm{eV}^{2}, \Delta_{41}=1.7 e V^{2}$ and mixing angles $\theta_{13}=10^{\circ}, \theta_{23}=45^{\circ}, \theta_{12}=34^{\circ}, \theta_{34}=$ $18.5^{\circ}, \theta_{24}=4^{o}, \theta_{14}=3.6^{\circ}$ 

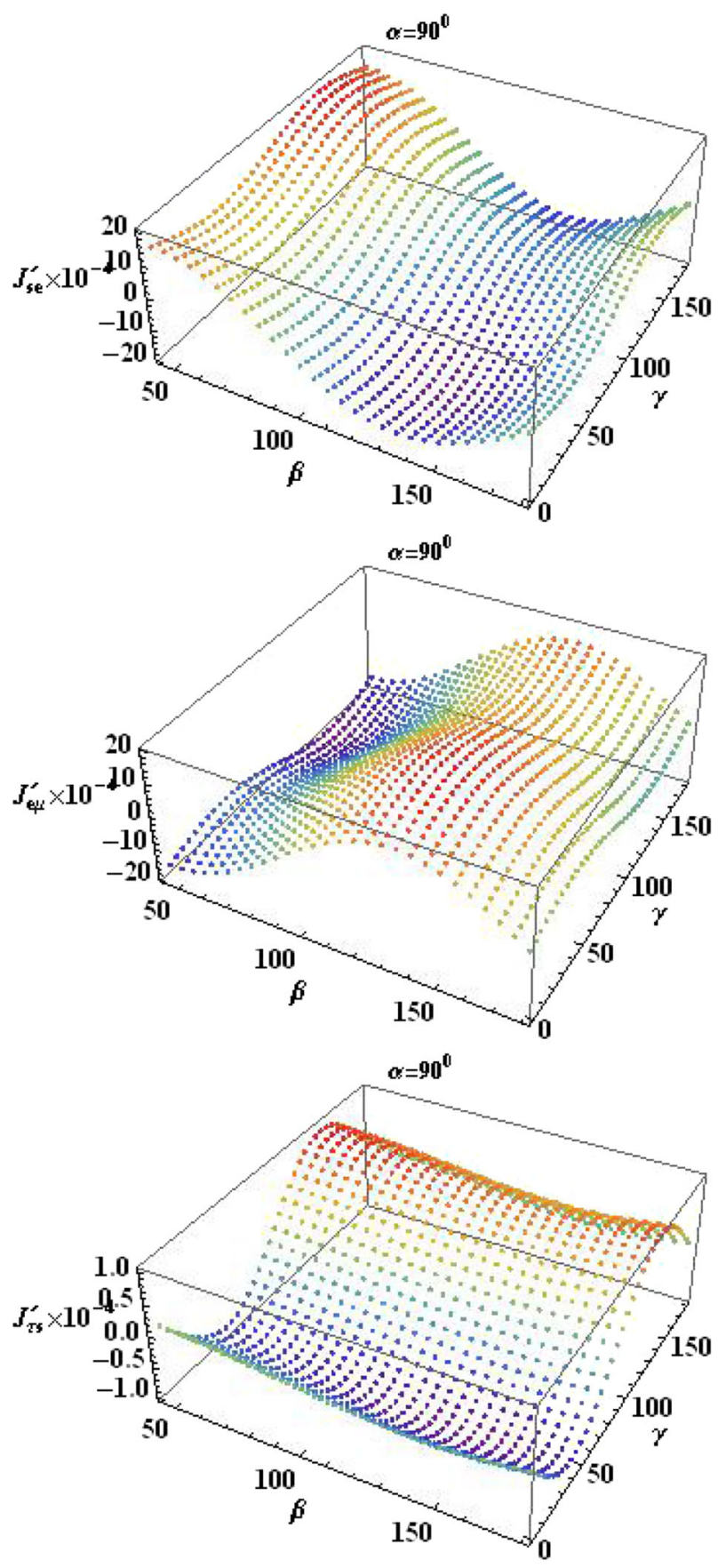

Fig. 3 For $\alpha=90^{\circ}$, the variation of $J_{s e}^{\prime}, J_{\tau s}^{\prime}$ and $J_{e \mu}^{\prime}$ with $\beta$ and $\gamma$. Input values are $\Delta_{31}=2.0 \times 10^{-3} \mathrm{eV}^{2}$, $\Delta_{21}=8.0 \times 10^{-5} \mathrm{eV}^{2}, \Delta_{41}=1.7 \mathrm{eV}^{2}$ and mixing angles $\theta_{13}=10^{\circ}, \theta_{23}=45^{\circ}, \theta_{12}=34^{\circ}, \theta_{34}=$ $18.5^{\circ}, \theta_{24}=4^{o}, \theta_{14}=3.6^{\circ}$ 

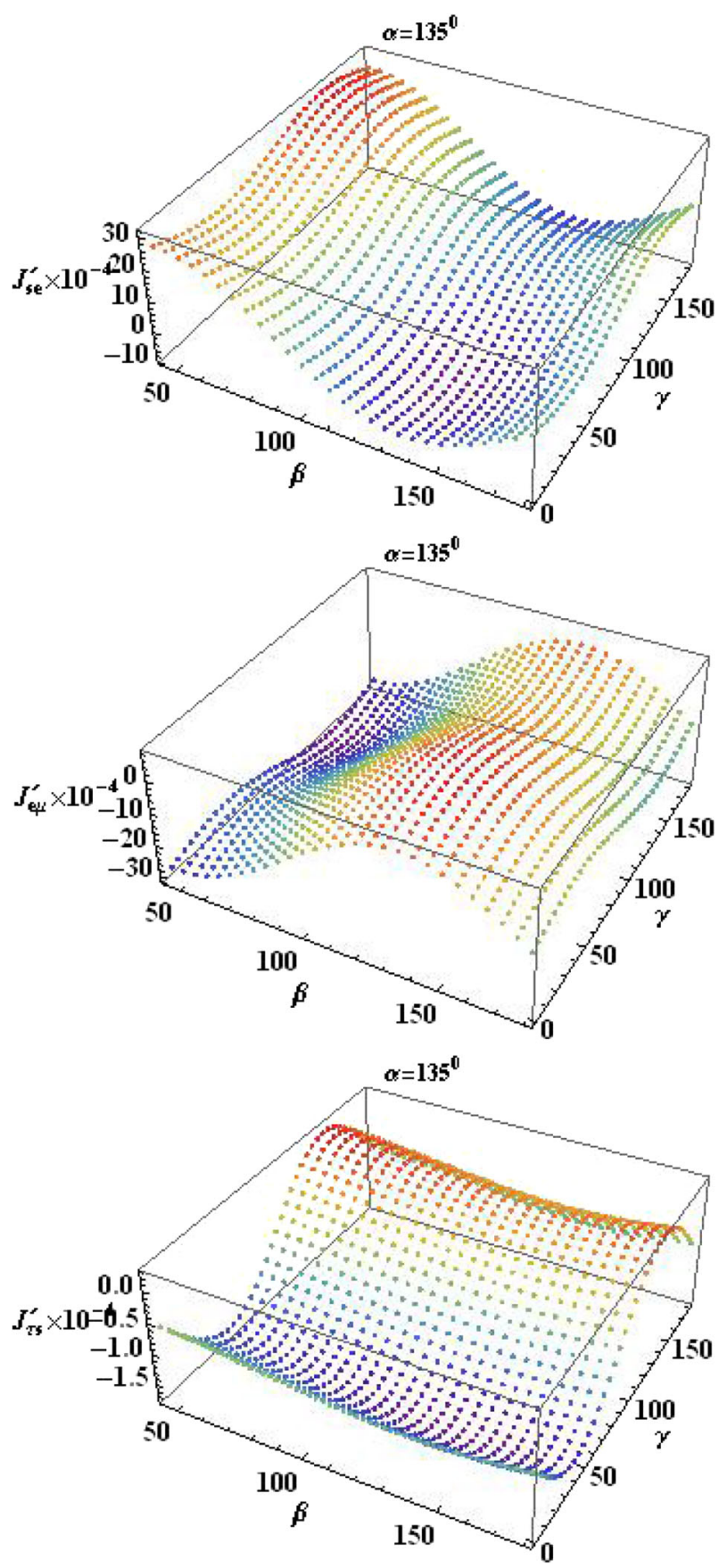

Fig. 4 For $\alpha=135^{\circ}$, the variation of $J_{s e}^{\prime}, J_{\tau s}^{\prime}$ and $J_{e \mu}^{\prime}$ with $\beta$ and $\gamma$. Input values are $\Delta_{31}=2.0 \times$ $10^{-3} \mathrm{eV}^{2}, \Delta_{21}=8.0 \times 10^{-5} \mathrm{eV}^{2}, \Delta_{41}=1.7 e V^{2}$ and mixing angles $\theta_{13}=10^{\circ}, \theta_{23}=45^{\circ}, \theta_{12}=34^{\circ}$ $, \theta_{34}=18.5^{\circ}, \theta_{24}=4^{\circ}, \theta_{14}=3.6^{\circ}$ 

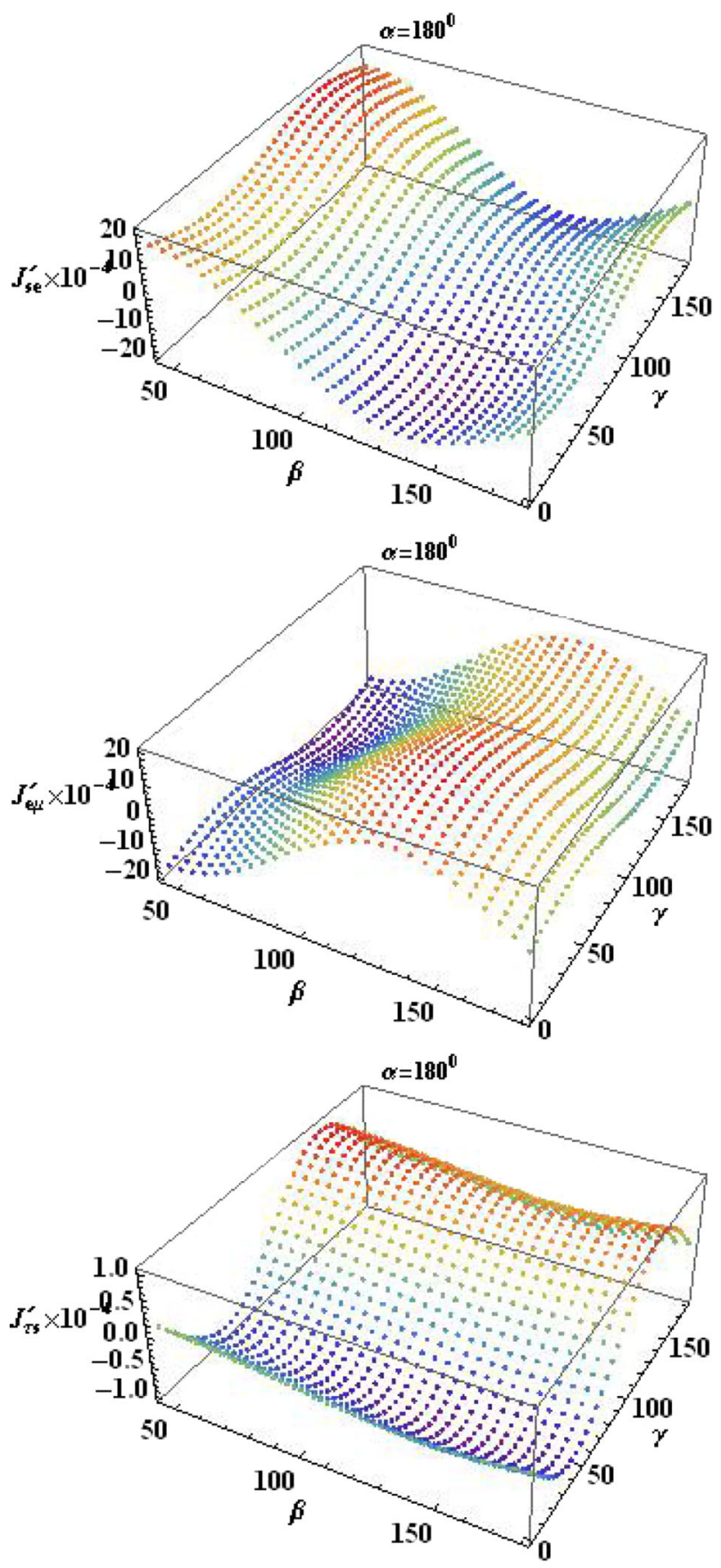

Fig. 5 For $\alpha=180^{\circ}$, the variation of $J_{s e}^{\prime}, J_{\tau s}^{\prime}$ and $J_{e \mu}^{\prime}$ with $\beta$ and $\gamma$. Input values are $\Delta_{31}=2.0 \times$ $10^{-3} \mathrm{eV}^{2}, \Delta_{21}=8.0 \times 10^{-5} \mathrm{eV}^{2}, \Delta_{41}=1.7 \mathrm{eV}^{2}$ and mixing angles $\theta_{13}=10^{\circ}, \theta_{23}=45^{\circ}, \theta_{12}=34^{\circ}$ $, \theta_{34}=18.5^{\circ}, \theta_{24}=4^{o}, \theta_{14}=3.6^{o}$ 
We know that the neutrino are almost degenerate just above the electroweak breaking scale. Therefore, we take a common neutrino mass to be $2 \mathrm{eV}$, which is upper limit of tritium beta decay spectrum [25]. The contribution of the term in beyond the GUT scale, $\epsilon=2\left(M_{i} \operatorname{Re}\left(m_{i i}\right)-M_{j} \operatorname{Re}\left(m_{j j}\right)\right.$, can be additive or subtractive depending on the values of the phase $\alpha, \beta, \gamma$ and phase $f_{i}$. We have taken $\Delta_{31}=0.002 e V^{2}[26,27]$, $\Delta_{21}=0.00008 \mathrm{eV}^{2}$ [28] and $\Delta_{41}=1.7 e \mathrm{~V}^{2}$ [29]. For simplicity, we have set the charge lepton phases $f_{1}=f_{2}=f_{3}=f_{4}=0$ and $\delta_{i j}=0$. The active sterile neutrino mixing angle are $\theta_{14}, \theta_{24}$ and $\theta_{34}$. In this work, we consider following value for sterile neutrino mixing angles [30], $\theta_{14}=3.6^{\circ}, \quad \theta_{24}=4^{\circ}, \quad \theta_{34}=18.5^{\circ}$. In Table 1 , we list combine value of Jarlskog Determinant above GUT scale for various value of Majorana phases $\alpha, \beta$ and $\gamma$. In Figs. 1, 2, 3, 4 and 5, we plot the Jarlskog Determinant with $\beta$ and $\gamma$ for different $\alpha$ values. As you can see from figures, the trend of graph is nearly same for different values of $\alpha$ but with different magnitudes of Jarlskog determinant which is in vertical axis.

\section{Conclusions}

The understanding of Jarlskog determinant is important for flavor physics. In both the quark and lepton sectors, this Jarlskog invariant provides the ability to control the magnitude of CP violation. We studied the Jarlskog determinant in four flavor framework above the GUT scale. We calculated the Jarlskog determinant by new MNS matrix in the form of $4 \times 4$ matrices, (19), by using four flavor mixing angle above the GUT scale, (2328 ) with current neutrino mixing angles. The values of Jarlskog determinant shows some phase inversion for some combinations of Majorana phases $\alpha, \beta$ and $\gamma$. Since, the magnitude of Jarlskog determinant above the GUT scale is of the order of $\sim 10^{-23}$. Hence, we tabulated the Jarlskog determinant which has much larger magnitude for some particular combination of Majorana phases $\alpha, \beta$ and $\gamma$. In the lepton sector above the GUT scale, we can constraint the Jarlskog determinant for normal mass order which is given as $-31.3015 \times 10^{-4} \leq J_{s e}^{\prime} \leq 31.3015 \times 10^{-4},-31.6378 \times 10^{-4} \leq J_{s e}^{\prime} \leq 31.6378 \times 10^{-4}$ and $-1.7739 \times 10^{-4} \leq J_{s e}^{\prime} \leq 1.7739 \times 10^{-4}$.

Open Access This article is licensed under a Creative Commons Attribution 4.0 International License, which permits use, sharing, adaptation, distribution and reproduction in any medium or format, as long as you give appropriate credit to the original author(s) and the source, provide a link to the Creative Commons licence, and indicate if changes were made. The images or other third party material in this article are included in the article's Creative Commons licence, unless indicated otherwise in a credit line to the material. If material is not included in the article's Creative Commons licence and your intended use is not permitted by statutory regulation or exceeds the permitted use, you will need to obtain permission directly from the copyright holder. To view a copy of this licence, visit http://creativecommons.org/licenses/by/4.0/.

\section{References}

1. Super-Kamiokande Collaboration, Fukuda, Y., et al.: Phys. Lett. B 436, 33 (1998)

2. Gariazzo, S., et al.: JHEP 1706, 135 (2017)

3. Gariazzo, S., et al.: J. Phys. G 43, 033001 (2016)

4. Super-Kamiokande Collaboration, Fukuda, Y., et al.: Phys. Rev. Lett. 81, 1562 (1998)

5. LSND Collaboration, Athanassopoulos, C., et al.: Phys. Rev. Lett. 81, 1774 (1998)

6. Bilenky, S.M., Giunti, C., Grimus, W., Schwetz, T.: Phys. Rev. D 60, 073007 (1999)

7. Jarlskog, C.: Phys. Rev. Lett. 55, 1039 (1985)

8. Kobayashi, M., Maskawa, T.: Theor. Prog. Phys. Rev. Lett. 45, 652 (1973) 
9. Verger, V.: Phys. Rev. Lett. 45, 2084 (1980)

10. Barger, V., Yuan-Ben Dai, K., Whisnant, Bing-Lin Young.: Phys. Rev. D. 59, 113010 (1999)

11. Dev, S., et al.: Nuclear. Phys. B 491, 401 (2019)

12. Dick, K., Freund, M., Lindner, M., Romanino, A.: Nucl.Phys.B 562, 29-56 (1999)

13. Kim, J.E., Kim, S.-J., Nam, S., Shim, M.: arXiv:1907.12247v3[hep-ph] (2020)

14. Wagner, D.J., Weiler, T.J.: arXiv:9801327[hep-ph] and arXiv:9806490[hep-ph] (1999)

15. Jarlskog, C.: Z. Phys. C29, 491 (1987). Phys. Rev. D35, 1685

16. Fritzsch, H., Xing, Z.Z.: Prog. Part. Nucl. Phys. 45, 1 (2000). and references therein

17. Koranga, B.S.: Int. J. Theor. Phys. 59, 3224-3228 (2020)

18. Koranga, B.S., Khurana, P.: Int. J. Theor. Phys. 53, 3737-3743 (2014)

19. Koranga, B.S., Uma Sankar, S.: EJTP 6(20), 229-234 (2009)

20. Koranga, B.S., Uma Sankara, S., Narayan, M.: Phys. Letts. B 665, 63-66 (2008)

21. Vissani, F., et al.: Phys.Lett. B571, 209 (2003)

22. Koranga, B.S., Prakash, G.: Int. J. Theor. Phys. 52, 2215-2223 (2013)

23. Koranga, B.S., Kumar, V., Jha, A.: Int. J. Theor. Phys. 50, 2609-2613 (2011)

24. Guo, W.-L., Xing, Z.-Z.: arXiv:0112121v2[hep-ph] (2001)

25. Kraus, Ch., et al.: Eur. Phys. J. C 40, 447-468 (2005)

26. Super-Kamiokande Collaboration, Hosaka, J., et al.: Phys. Rev. D 74, 32002 (2006)

27. T2K Collaboration, Abe, K., et al.: Phys. Rev. Lett. 112, 181801 (2014)

28. KamLAND Collaboration, Hosaka, J., et al.: Phys. Rev. Lett. 94, 08180 (2005)

29. MINOS Collaboration, Adsmson, P., et al.: Phys. Rev. D 072002, 77 (2008)

30. Pandey, M., et al.: arXiv:2003.02102[hep-ph] (2020)

31. Koranga, B.S., Nautiyal, V.K., Jha, A.K., Narayan, M.: Int. J. Theor. Phys. https://doi.org/10.1007/ s10773-021-04811-2 (2021)

Publisher's Note Springer Nature remains neutral with regard to jurisdictional claims in published maps and institutional affiliations. 